\title{
Design of a K/Q-band Beacon Receiver for the Alphasat TDP\#5 Experiment
}

\author{
James A. Nessel, Michael J. Zemba, Jacquelynne R. Morse \\ Antenna and Optical Systems Branch \\ NASA Glenn Research Center \\ Cleveland, OH, USA \\ jacquelynne.r.morse@nasa.gov
}

\begin{abstract}
This paper describes the design and performance of a coherent $\mathrm{K} / \mathrm{Q}$-band $(20 / 40 \mathrm{GHz})$ beacon receiver developed at NASA Glenn Research Center (GRC) that will be installed at the Politecnico di Milano (POLIMI) for use in the Alphasat Technology Demonstration Payload \#5 (TDP\#5) beacon experiment. The goal of this experiment is to characterize rain fade attenuation at $40 \mathrm{GHz}$ to improve the performance of existing statistical rain attenuation models in the Q-band. The ground terminal developed by NASA GRC utilizes an FFT-based frequency estimation receiver capable of characterizing total path attenuation effects due to gaseous absorption, clouds, rain, and scintillation. The receiver system has been characterized in the lab and demonstrates a system dynamic range performance of better than $58 \mathrm{~dB}$ at $1 \mathrm{~Hz}$ and better than $48 \mathrm{~dB}$ at $10 \mathrm{~Hz}$ rates.
\end{abstract}

\section{INTRODUCTION}

Though the primary focus of NASA's future space communications architecture is in the exploitation of $\mathrm{Ka}$ and optical frequencies, NASA is also investigating the potential use of spectrum in the Ka/Q-bands $(37-42 \mathrm{GHz})$ and $\mathrm{V} / \mathrm{W}$ bands $(74-84 \mathrm{GHz})$ as a downlink option in the next generation Space Based Relay (SBR) that is expected to replace the existing Tracking and Data Relay Satellite (TDRS) system in the 2025 timeframe. As such, NASA Glenn Research Center is spearheading a small, collaborative effort to characterize atmospheric propagation effects at these millimeter wave frequencies. A major part of this campaign includes the use of the Alphasat TDP\#5 20/40GHz beacon in Europe. The primary goal of these measurements is to develop a physical model to improve predictions of communications systems performance within the desired spectrum. Herein, we describe the design and laboratory measured performance of the $20 / 40 \mathrm{GHz}$ beacon receiver built in-house by NASA Glenn Research Center, to be installed at the Politecnico di Milano in Milan, Italy. The receiver is based upon the validated Fast Fourier Transform (FFT) digital design approach utilized in other operational GRC propagation terminals [1-2]. The new design incorporates upgrades and modifications to coherently track and measure the amplitude of the $20 / 40 \mathrm{GHz}$ beacon signals with improved dynamic range. It is believed that these measurements, combined with thorough characterization of local meteorological conditions, will provide the necessary basis for a preliminary model to assess the performance of communications systems employing frequencies in the $\mathrm{Q}$, as well as $\mathrm{V} / \mathrm{W}$-bands.

\section{GRC K/Q-BAND BEACON RECEIVER DESIGN}

The beacon receiver developed at NASA GRC consists of a $1.2 \mathrm{~m}$ K-band and a $0.6 \mathrm{~m}$ Q-band Cassegrain reflector employing independent open-loop tracking systems for each antenna to track the inclined orbit of the Alphasat satellite. A block diagram of the front ends and common Intermediate Frequency (IF) components of the system is provided in Figure 1 and a photograph of the final hardware is shown in Figure 2. Down_conversion of the $19.701 \mathrm{GHz}$ and $39.402 \mathrm{GHz}$ signals broadcasted from Alphasat is performed in multiple stages to a common IF of $455 \mathrm{kHz}$. All local oscillators (LO's) utilize a common ultra-stable $10 \mathrm{MHz}$ reference oscillator to maintain coherency.

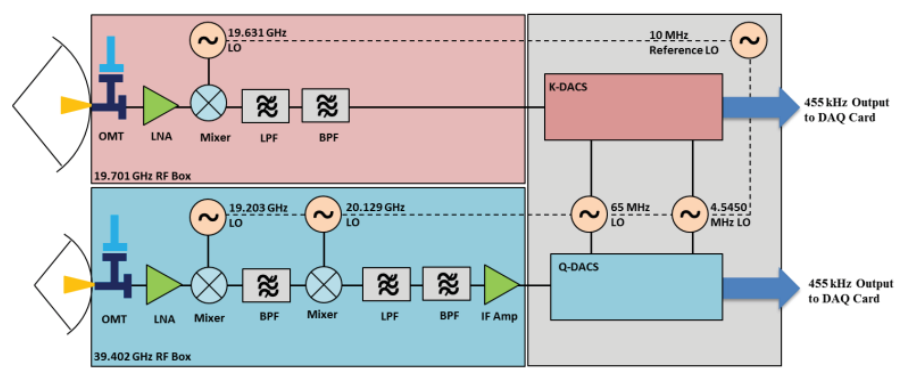

Figure 1. Block diagram of coherent K/Q-band beacon receiver

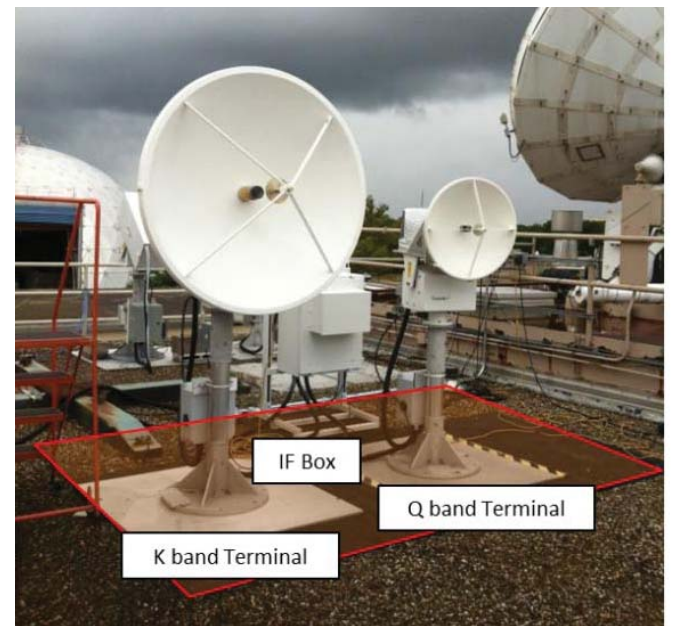

Figure 2. Photograph of K-band and Q-band beacon receiver system at NASA GRC's outdoor test site. 
The $455 \mathrm{kHz}$ IF signal is undersampled by a National Instruments 5124 DAQ card at a sampling frequency (fs) of $111.111 \mathrm{kHz}$. At a $1 \mathrm{~Hz}$ data measurement rate, we collect $2^{16}$ samples for a final $\mathrm{fs} / \mathrm{N}$ resolution of $1.7 \mathrm{~Hz}$. Frequency estimation and power measurement of the beacon signal employs a modified Quinn-Fernandes frequency estimation routine, as described in [3]. During deep rain fades, i.e., fades greater than $40 \mathrm{~dB}$, the beacon receiver utilizes the K-band signal for frequency tracking to maintain lock on the Q-band signal. Based on this approach, the measurement range of the Q-band receiver is improved down to a $3 \mathrm{~dB}$ bin signal to noise ratio (SNR) and is capable of reestablishing lock once the signal immerges from the noise. Figure 3 shows the estimated frequency bin offset that is used by the modified QuinnFernandes algorithm to determine the spectral location of the Q-band signal during deep fades.

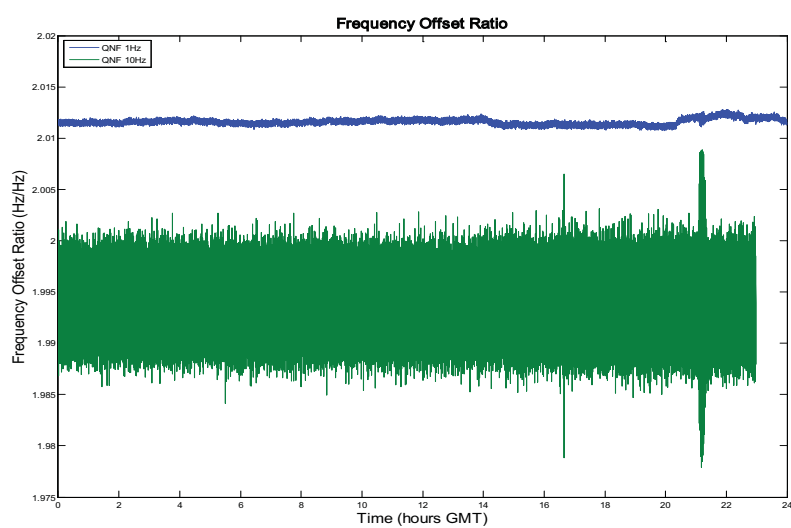

Figure 3. Frequency bin offset between the K- and Q-band signals. This information is used to maintain lock

\section{GRC K/Q-BAND BEACON RECEIVER PERFORMANCE}

The system noise temperature of the K-band and Q-band receivers were measured to be approximately $504 \mathrm{~K}$ and $720 \mathrm{~K}$, respectively. Based on the expected beacon signal levels from the Alphasat payload and propagation path, a link budget was developed and indicates a clear sky receive carrier-to-noise density $\left(\mathrm{C} / \mathrm{N}_{0}\right)$ of $55 \mathrm{~dB}-\mathrm{Hz}$ for both systems.
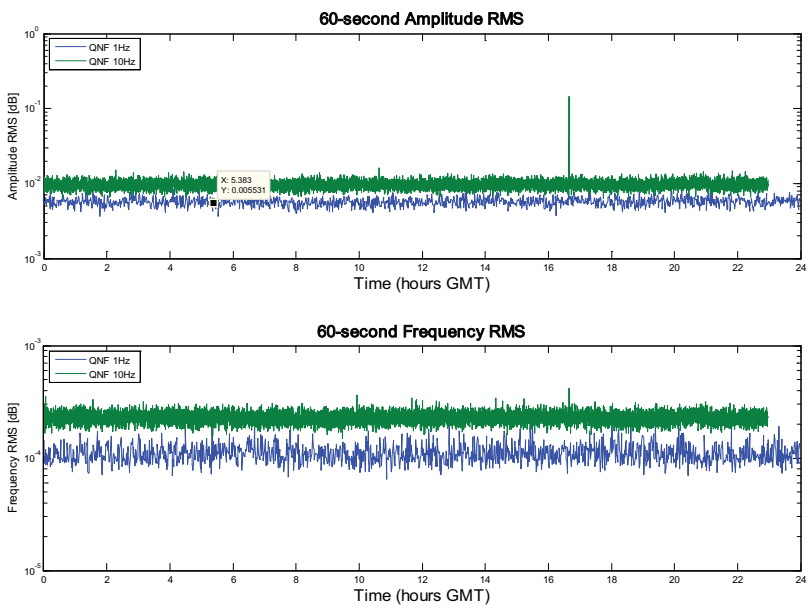

Figure 4. Amplitude and frequency stability of the Q-band receiver
Nominal operation of the receiver records data at a $1 \mathrm{~Hz}$ rate. However, to characterize scintillation performance an output sample rate of greater than that current $1 \mathrm{~Hz}$ is required [4]. Accordingly, the receiver software was modified to adjust the measurement rate to $10 \mathrm{~Hz}$ to capture scintillation effects, on demand. Figure 4 shows the system performance in clear sky conditions. The measurement resolution of the system during this condition is $.009 \mathrm{~dB}$ root-mean-square (RMS) at $10 \mathrm{~Hz}$ and $.005 \mathrm{~dB}$ RMS at $1 \mathrm{~Hz}$.

Increasing the measurement rate requires reducing the number of samples from $2^{16}$ to $2^{13}$ to reduce integration time below $100 \mathrm{~ms}$. This results in a noise floor increase of approximately $10 \mathrm{~dB}$ when scintillation information is desired. Figure 5 depicts the increase in the noise floor of the $10 \mathrm{~Hz}$ system.

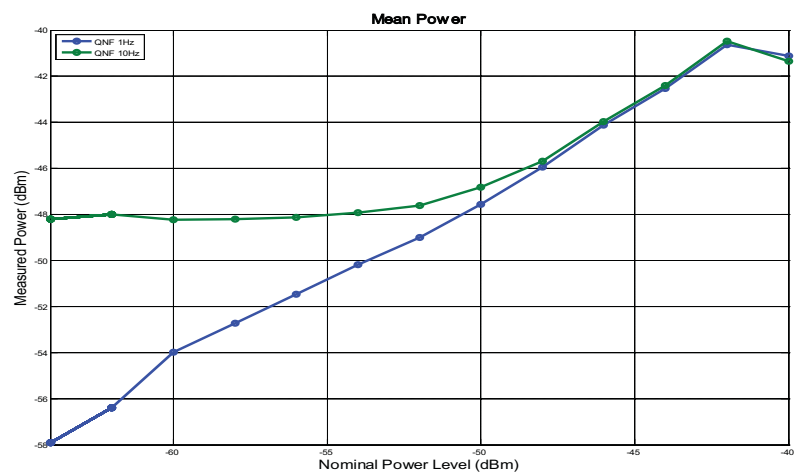

Figure 5. Noise floor performance of the Q-band receiver system at both the 10 and $1 \mathrm{~Hz}$ output rate

\section{CONCLUSIONS}

The design and performance of the GRC-developed K/Qband beacon receiver developed for the Alphasat TDP\#5 experiment has been validated in the laboratory and has been shown to meet the requirements of the propagation experiment. Delivery and installation of the receiver at the Politecnico di Milano is planned for March 2014. The measurement campaign is expected to last for a minimum of 3 years, with potential for up to 5 years, which represents an acceptable amount of time to observe a full weather cycle [5] at a location and to have a statistically relevant data set.

\section{REFERENCES}

[1] R. Acosta, et. al., "Goldstone Site Test Interferometer," $13^{\text {th }} \mathrm{Ka}$ and Broadband Comm. Conference, Turin, Italy, Sep. 2007.

[2] R. Acosta and J. Nessel, "Data Processing for Atmopheric Phase Interferometers", NASA TM 2009-215599

[3] M. Zemba et al., "Frequency Estimator Performance for a SoftwareBased Beacon Receiver," In Proceedings of the 2014 IEEE Antennas and Propagation Conference, July 6-12, 2014, Memphis, TN.

[4] Singh et al. "Measurement of Tropospheric Scintillation from Satellite Beacon at Ku-Band In South East Asia", IJCSNS International Journal of Computer Science and Network Security, VOL.7 No.2, February 2007

[5] A. R. Thompson et al. "Interfermoetry and Synthesis in Radio Astromomy", $2^{\text {nd }}$ ed, New York, Wiley, 2001 